\title{
As conexões entre Sustentabilidade e Desenvolvimento Sustentável com a Demografia Espacial
}

\author{
Karina M. Berbert Bruno ${ }^{1}$ \\ Ana Cláudia dos Santos Luciano ${ }^{2}$
}

Resumo: A interação entre População-Ambiente foi abordada por Daniel Joseph Hogan em sua pesquisa, projetos e publicações, promovendo através deles a integração de diferentes áreas do conhecimento. Esta integração se baseou, principalmente, na utilização de abordagens e análises demográficas, geográficas-espaciais e sociológicas. A interação entre espaço geográfico e aspectos demográficos tais como expansão urbana e migração são discutidas neste trabalho, de acordo com as proposições de Hogan. No presente artigo, as contribuições do autor sobre a relação existente entre os estudos demográficos com os conceitos de Sustentabilidade e Desenvolvimento Sustentável são analisadas conjuntamente com a componente do espaço geográfico. Tais contribuições são resgatadas e trazidas para o debate atual, visando dialogar com o campo chamado de Demografia Espacial. Dessa forma, iniciativas e estudos desenvolvidos até então que contemplaram de forma bem-sucedida ou não tal conexão entre Sustentabilidade, Desenvolvimento Sustentável e a Demografia Espacial são exemplificados neste artigo. Neste sentido, destaca-se também os estudos de Hogan a respeito dos temas de Sustentabilidade e Desenvolvimento Sustentável que nos trazem os conceitos de Capacidade de Suporte e a possibilidade de considerar nos estudos demográficos, as diferentes escalas de análises e os seus respectivos contextos espaciais. Por fim, aqui é proposta a consideração de que a Sustentabilidade e o Desenvolvimento Sustentável são conceitos dinâmicos e que dependem do momento histórico, político, social, econômico e geográfico em que estão inseridos. Esta consideração pode, ainda, ser um caminho para políticas públicas e ações efetivas na preservação ambiental, equidade social e eficiência econômica.

Palavras-chave: Ecossistemas. Dinâmica demográfica e socioespacial. Espaço Geográfico.

1 Graduada em Geografia (Instituto de Geociências) pela UNICAMP e mestranda em Demografia no Instituo de Filosofia e Ciências Humanas, linha População e Ambiente pela UNICAMP. E-mail: k139566@dac.unicamp.br

${ }^{2}$ Doutora pela Faculdade de Engenharia Agrícola da UNICAMP. Professora da Escola Superior de Agricultura Luiz de Queiroz (ESALQ/USP). Piracicaba, SP. E-mail: analuciano@usp.br 


\title{
Connections between Sustainability and Sustainable Development with Spatial Demography
}

\begin{abstract}
The integration between different knowledge fields was proposed by the Population-Environment analysis, addressed by Daniel Joseph Hogan in his research, projects, and publications. This integration was mostly based on spatial, demographic, and sociological approaches and analyses. The geographic space and demographic aspects interactions such as urban sprawl and migration are discussed in this work, according to Hogan's contributions. In the author's research, the relationship between demographic studies, Sustainability, and Sustainable Development concepts is analyzed with the space component. This approach is discussed in the current time, also communicating with the field of Spatial Demography. Initiatives and studies involving a well-predicted result or not of such connection of Sustainability, Sustainable Development, and geographic space are also considered. In this sense, Hogan's studies on the themes of Sustainability and Sustainable Development also bring us the concepts of Carrying Capacity and the possibility of considering demographic studies in different scales of analysis and their respective spatial contexts. Finally, it is proposed the consideration of Sustainability and Sustainable Development as dynamic concepts, depending on their historical, political, social, and economic frame, as well as their geographic location. This consideration can be a path for effective policies and actions for environmental preservation, social equity, and economic efficiency.
\end{abstract}

Keywords: Ecosystems. Demographic and socio-spatial dynamics. Geographical space.

\section{Las conexiones entre la Sostenibilidad y el Desarrollo Sostenible con la Demografía Espacial}

Resumen: La interacción entre Población-Medio Ambiente fue abordada por Daniel
Joseph Hogan en sus investigaciones, proyectos y publicaciones, promoviendo a través
de ellos la integración de diferentes áreas del conocimiento. Esta integración se basó
principalmente en el uso de enfoques y análisis demográficos, geográfico-espaciales y
sociológicos. La interacción entre el espacio geográfico y aspectos demográficos como
la expansión urbana y la migración se discuten en este trabajo, según las contribuciones
de Hogan. En este artículo se analizan las aportaciones del autor sobre la relación entre
los estudios demográficos y los conceptos de Sostenibilidad y Desarrollo Sostenible
junto con el componente de espacio geográfico. Tales aportaciones son rescatadas
y llevadas al debate actual, con el objetivo de dialogar con el campo denominado
Demografía Espacial. De esta forma, las iniciativas y estudios desarrollados hasta el
momento que contemplaron con éxito o sin éxito tal conexión entre Sostenibilidad,
Desarrollo Sostenible y Demografía Espacial se ejemplifican en este artículo. En este 
sentido, también se destacan los estudios de Hogan sobre los temas de Sostenibilidad y Desarrollo Sostenible, que nos acercan los conceptos de Capacidad de Carga y la posibilidad de considerar, en estudios demográficos, las diferentes escalas de análisis y sus respectivos contextos espaciales. Finalmente, aquí se propone considerar que Sostenibilidad y Desarrollo Sostenible son conceptos dinámicos que dependen del momento histórico, político, social, económico y geográfico en el que se insertan. Esta consideración también puede ser un camino para políticas públicas y acciones efectivas en preservación ambiental, equidad social y eficiencia económica.

Palabras clave: Ecosistemas. Dinámica demográfica y socioespacial. Espacio geográfico.

\section{Introdução}

O termo Sustentabilidade apresenta uma longa e complexa história de conceituação e aplicação. A sua primeira utilização na literatura data de 1972, em um livro britânico que abordava questões e preocupações referentes ao futuro da humanidade (KIDD, 1992). De fato, ao se realizar o estudo e análise de referências bibliográficas conceituais, observa-se que o conceito de Sustentabilidade é aprofundado, expandido e modificado por diversos agentes e instituições de forma desconexa. Esta indefinição conceitual leva, consequentemente, a práticas diversas ditas sustentáveis, sendo que em muitos casos não são, necessariamente, benéficas nas áreas ambiental, social e política, tendo-se como resultado diferentes frentes de atuação baseadas neste conceito. Mas afinal, o que seria então a Sustentabilidade?

Desde a primeira aplicação do termo até os dias atuais, a Sustentabilidade é colocada em conexão com as esferas ambientais, econômicas, políticas e sociais. Inicialmente, pode-se dizer que o foco conceitual da Sustentabilidade se deu, principalmente, sobre a capacidade efetiva dos recursos naturais suprirem as demandas de consumo das populações ao redor do globo (BRUNDTLAND, 1987; KIDD, 1992; RATTNER, 1999). Com o avanço das pesquisas científicas e os avanços tecnológicos, proposições determinísticas referentes à pressão destrutiva do ser humano sobre meio ambiente foram superadas e o conceito de Sustentabilidade passou a ser utilizado e compreendido de forma mais abrangente. 
$|4|$

As conexões entre Sustentabilidade e Desenvolvimento...

Atualmente, além do conceito vincular-se ao uso eficiente dos recursos naturais, ele representaria, essencialmente, a relação equilibrada entre sociedade, população e ambiente. Entendese que diretrizes, projetos, iniciativas públicas ou privadas que se baseiem na Sustentabilidade devem direcionar intervenções através de ações individuais, coletivas, sociais e governamentais no atual sistema econômico produtivo e de consumo, proporcionando a preservação dos ecossistemas e qualidade de vida para os indivíduos (JOHNSTON et. al., 2007; KUHLMAN, FARRINGTON, 2010; MORELLI, 2011).

Hogan (1993) nos leva a refletir em seu artigo intitulado "Crescimento populacional e Desenvolvimento Sustentável" se existiria a possibilidade da elaboração de conceitos concretos a respeito dos temas Sustentabilidade e Desenvolvimento Sustentável (DS). Seria possível um conceito robusto e bem definido destes temas serem colocados como norteadores para políticas, governos e sociedades? Esta reflexão surge, principalmente, porque o autor destaca que nas sociedades contemporâneas, tais temas se tornaram um "lugar-comum", requeridos e proferidos por muitas pessoas, organizações e governos, sem necessariamente se basearem nas mesmas diretrizes e aplicações, tornando-se, portanto, um desafio encontrar uma agenda de Sustentabilidade comum a todos.

Neste sentido, a proposta de Rattner (1999) afirma que o mais importante avanço na evolução do conceito de Sustentabilidade é representado pelo consenso crescente de que a Sustentabilidade exige per se a proteção e conservação do meio ambiente, democracia política, equidade social, eficiência econômica e diversidade cultural, e que esta síntese, ainda que não aceita por todos, tenderá a exercer uma influência poderosa na teoria e na prática social nos anos futuros. Tal definição é o que se tem visto nos últimos anos, principalmente em diretrizes sustentáveis internacionais como os Objetivos de Desenvolvimento Sustentáveis da ONU (ODS) (ONU, 2015) e as diretrizes da Agenda 2030 para o Desenvolvimento Sustentável (ONU, 2021).

Sobre o Desenvolvimento Sustentável (DS), segundo Sartori et al. (2014), este seria uma elaboração conceitual chave que tem 
como base os princípios da Sustentabilidade. Tais princípios seriam: a perspectiva de longo prazo do uso e manutenção dos recursos naturais, a importância fundamental das condições ambientais locais e a compreensão da evolução não linear dos sistemas ambientais e humanos. Ambos os conceitos de Sustentabilidade e DS se mostram em constante dinâmica de aplicação, adequandose de acordo com a conjuntura do momento histórico, geográfico e político. Certos parâmetros, vinculados a estes temas se mostram consolidados, principalmente aqueles difundidos por organizações internacionais como a ONU, sendo os principais deles a preservação ambiental, equidade social e eficiência econômica. Nota-se, portanto, que debates e discussões acerca da Sustentabilidade na literatura e no campo político governamental vem associada com a temática do DS e, outros fatores (por exemplo, espaço geográfico) passam a ser colocados em destaque.

\subsection{A componente espacial: relações sociais, econômicas e demográficas com o ambiente}

A componente espacial tem sido colocada como um dos fatores determinantes para se pensar em Sustentabilidade e DS devido aos diversos desafios ambientais, econômicos e sociais que variam entre escalas local, regional, nacional e global. Além disso, somam-se as questões referentes à localização e disponibilidade de recursos naturais; biodiversidade; fatores demográficos (densidade populacional, centros de consumo), dentre outros (DE CASTRO, 2007).

Martine (2007) exemplifica essa associação de Sustentabilidade, DS e a componente espacial em seu texto "O lugar do espaço na equação população/meio ambiente". O autor nos mostra que o DS é um fator determinante no que se refere aos assuntos ambientais, destacando que a Sustentabilidade vem definir no DS não somente os padrões de produção e consumo de um dado território, mas também a alocação espacial das atividades econômicas. $\mathrm{Ou}$ seja, a Sustentabilidade passa a direcionar as práticas do DS não só nas esferas econômicas, políticas e sociais, mas também, espaciais. 
$|6|$

As conexões entre Sustentabilidade e Desenvolvimento..

A dimensão espacial da sociedade está, portanto, intrinsecamente relacionada com todo o seu desenvolvimento, seja ele econômico, social, político etc., influenciando, por exemplo, na capacidade de oferta de bens e serviços, na capacidade de consumo e no acesso à infraestrutura proporcionada por esferas governamentais (SANTOS, 1996). Ou seja, diferentes espaços geográficos podem fornecer variados meios de produção, transporte, mão de obra, recursos naturais, dentre outros aspectos. A sociedade e, consequentemente, as dinâmicas populacionais a ela vinculadas permeiam, moldam e são influenciadas pelo espaço (SANTOS, 1996). Caso o objetivo de uma sociedade ou entidades governamentais seja aplicar práticas que promovam o desenvolvimento social e econômico com diretrizes sustentáveis, torna-se, portanto, necessário articular processos e agentes de naturezas distintas em diferentes escalas espaciais. Dessa forma, os espaços geográficos tornam-se agentes inseparáveis dos conceitos de Sustentabilidade e DS.

Deve-se, portanto, evitar analisar qualquer frente de desenvolvimento apartada de seu contexto geográfico espacial. Neste sentido, Hogan fez importantes contribuições ao incorporar a dimensão espacial em análises vinculadas aos temas de Sustentabilidade, DS e estudos demográficos, trazendo a componente espacial para a relação População-Ambiente (P-A).

Para a análise da contribuição pioneira de Hogan sobre a interação entre Sustentabilidade, DS, estudos demográficos e dimensão espacial, será definido primeiramente o conceito de espaço geográfico no presente trabalho, e destacadas as proposições de outros autores sobre a demografia espacial. Por espaço geográfico, entende-se que: o conceito não se refere estritamente ao espaço físico-descritivo, mas sim, a um conjunto de configurações espaciais e dinâmicas sociais (SANTOS, 1996).

Seguindo nesta proposição e envolvendo toda a complexidade deste conceito, Hogan (1991) e Voss (2007) enfatizam ainda a relação existente entre dinâmica populacional e espaço geográfico, ou, processos sociais em um contexto espacial. Tal relação seria contemplada pelo campo da demografia espacial. Sobre isto, 
Martine (1993) contribuiu para a construção do campo de estudo da Demografia Espacial, explicitando que a dimensão demográfica apresenta padrões e comportamentos diversos em diferentes escalas espaciais, regiões e países, sendo que questões vinculadas à esfera ambiental também variam em escala. Nesse sentido, o autor vincula análises de viés econômico, como padrões de produção e consumo com dinâmicas populacionais e a componente espacial. Castro (2007) propõe a reflexão de que a componente espacial tem uma função descritiva, capturando, preservando e transmitindo as aparências e relações econômicas, sociais e demográficas observadas entre objetos em um dado espaço. Desta forma, a autora destaca que o uso de métodos analíticos espaciais na pesquisa demográfica representa um passo adiante na proposição de políticas mais realistas e adequadas ao ambiente e populações a que se destinam. Partindo desta correlação entre dinâmicas populacionais, econômicas e o espaço geográfico pode-se definir a demografia espacial.

A demografia espacial é uma área interdisciplinar dentro do campo da demografia, que colabora para a compreensão das relações entre conjunturas históricas, sociais, ambientais, econômicas e espaciais. Temas importantes como Sustentabilidade e DS em uma dada região ou país recebem, dentro da demografia espacial, a correlação com seus respectivos contextos e especificações históricas, socioeconômicas e socioespaciais.

Desta forma, para compreender a influência do espaço em questões sociais, demográficas, econômicas, ambientais e outras, a proposta de Marandola Jr. e Hogan (2007), considera dois fatores de análise: 1) a distribuição da população e 2) a dinâmica migratória em um dado território. Um exemplo claro disto é apontado por Hogan et al. (2008), em um estudo sobre a dinâmica demográfica da Amazônia. Os autores destacam que as diferenças regionais e intrarregionais das formas de ocupação ocorridas na Amazônia originaram-se de diferentes estratégias ou objetivos de povoamento (mineração, extrativismo vegetal, agropecuária, dentre outros), o que resultou em diferentes formas de organização territorial. Além disso, as variações dos fluxos migratórios e 
$|8|$

As conexões entre Sustentabilidade e Desenvolvimento...

povoamento que ocorreram nesta região, foram incentivadas por esforços do Estado em diferentes períodos no decorrer do tempo. Observa-se claramente neste exemplo a dinâmica existente entre fatores sociais e ambientais; dinâmicas demográficas e espaciais; e, por último, a influência governamental e política em todos estes aspectos ao mesmo tempo.

Com base na proposta de Marandola Jr. e Hogan (2007) e no exemplo destacado por Hogan et al. (2008) é possível visualizar a interação entre dinâmica populacional e espaço geográfico, ou seja, é possível analisar os processos sociais e demográficos em um contexto espacial - a demografia espacial. De acordo com Hogan et al. (2008), a relação entre população, ambiente e sociedade é explicitada por fatores migratórios, disponibilidade de recursos naturais e preferências políticas de desenvolvimento econômico. Como poderíamos então, estruturar a aplicação de DS baseado nas diretrizes da Sustentabilidade, uma vez que dinâmicas populacionais, econômicas, sociais e políticas estão vinculadas ao espaço?

Para isto seria necessário então, passar a elaborar as práticas de Sustentabilidade e DS de forma regionalizada, considerando as características, capacidades e limitações de cada espaço geográfico, ou seja, as particularidades econômicas, sociais e ambientais. Devemos destacar que, no caso de países em desenvolvimento, como é o caso do Brasil, a Sustentabilidade e o DS permanecem sob a égide do neoliberalismo e, por isso, são colocados em prática de forma majoritariamente incompatível com qualquer definição ou orientação conceitual destes temas.

Para o Brasil, tal fato se justifica, principalmente, devido às assimetrias estruturais de poder no país, as quais subsidiam interesses de determinados grupos em detrimento do interesse geral da sociedade brasileira. Como consequência dessa assimetria de poder, grupos dominantes delimitam e reconfiguram as instituições e políticas referentes às frentes ambientais e sociais da forma que melhor convém aos ditames do mercado financeiro e econômico - e também aos seus próprios interesses individuais (GONÇALVES, 2019). Disto decorre a flexibilização das leis e 
desmantelamento das instituições de fiscalização e preservação ambientais que atualmente vemos no país, favorecendo, por exemplo, a expansão de práticas agrícolas e de mineração em biomas considerados ameaçados de degradação ambiental e classificados como hot spots de biodiversidade do planeta (KLINK, MACHADO, 2005).

Sendo assim, após o esclarecimento e exemplificação da relação entre dinâmicas populacionais, espaço geográfico e sociedade que podem ser abordadas pela demografia espacial, devese considerar os múltiplos sentidos e aplicações que preenchem o lugar-comum da Sustentabilidade e do DS destacados por Hogan (1993), o que torna complexa a relação entre a demografia espacial e estes temas. É necessário analisar a Sustentabilidade e DS nos seus respectivos seios estruturais históricos, políticos, econômicos, sociais, culturais e geográficos. Cada sociedade apresenta uma conjuntura de desenvolvimento histórico, socioeconômico e geográfico específico, sendo impraticável, por exemplo, replicar de forma cristalizada os modelos de Sustentabilidade e DS norteamericanos, saxônicos, nipônicos ou europeus de um modo geral no Brasil ou nos demais países sul-americanos.

Tal conjuntura histórica, social, econômica e espacial vinculase ainda, como destacado por Hogan (1993), às forças culturais centradas nos valores ocidentais do individualismo, do significado da vida e do que proporciona a satisfação ao ser humano, sendo que estas questões estão frequentemente associadas aos temas e debates da Sustentabilidade DS. O autor aponta que, em particular, o conceito de necessidades humanas (básicas/não-básicas e materiais/não materiais) deve ser considerado e requer elaboração e uma ampla discussão para poder redirecionar os esforços de desenvolvimento socioeconômico. Desta forma, as necessidades de indivíduos e sociedades exercem sobre o ambiente e recursos naturais uma pressão cada vez mais aniquiladora, lembrando que não se trata de uma pressão igualmente distribuída, ou seja, não ocorre um consumo individual homogêneo dos recursos do planeta, não dependendo unicamente do aumento ou diminuição da população, uma vez que que menos de $20 \%$ da população 
mundial consome mais de $80 \%$ dos recursos naturais do planeta (INMETRO/IDEC, 2002).

O resultado seria, portanto, um entendimento e valorização dos conceitos e práticas da Sustentabilidade e DS heterogêneos por diferentes sociedades, corroborando com a conceitualização feita por Hogan (1993) para lugar-comum e com as proposições sobre o entendimento geral de Sustentabilidade até aqui discutidos. O primeiro passo para pensarmos em uma agenda de Sustentabilidade e DS que seja compatível em diferentes escalas, populações, ambientes e sociedades talvez seja reconhecer que ela deverá sim ter um núcleo consolidado de diretrizes, mas que a sua operabilidade deverá ser adaptada a cada contexto espacial, econômico e social.

\section{Sustentabilidade e Desenvolvimento Sustentável com base na Demografia Espacial}

Buscando partir para uma análise prática da Sustentabilidade e DS, ou seja, uma análise que direcione para ações efetivas, concretas e, consequentemente, que provoquem a alteração da realidade existente, pode-se utilizar como norteadoras as palavras de Hogan (1993): capacidade de suporte (CS) e ecossistema.

Sobre a capacidade de suporte, Hogan (1993) destaca que foi através deste conceito que a preocupação com o DS se exprimia no campo da demografia, podendo ser inicialmente bem explicado pela definição proposta pela UNESCO e utilizada por Hogan (1993):

A capacidade de suporte expressa o nível de população que pode ser sustentado por um país em um dado nível de bem-estar. Mais precisamente, ela pode ser definida como o número de pessoas compartilhando um dado território que podem sustentar, de uma forma que seja viável no futuro, um dado padrão material de vida utilizando-se de energia e de outros... Nunca há uma solução única para a equação população/ recursos naturais, pois não é somente a população que 
determina a pressão sobre os recursos (e os potenciais efeitos ecológicos associados) mas também o consumo individual que, por sua vez, é determinado pelo sistema de valores e pelas percepções de estilo de vida.

Para o autor uma definição abrangente e satisfatória do conceito de CS seria a inclusão não apenas do abastecimento alimentício, mas também de outras necessidades humanas básicas e não-básicas. Isto implica em: 1) considerar critérios culturais de determinados povos e regiões e, 2) admitir o aumento ou a redução da capacidade de suporte através de: progresso tecnológico, aumento da igualdade social, aumento dos níveis de educação e saúde, descobertas de novos recursos minerais e forças políticas ou ideológicas. Desta forma, o autor conclui que seria possível mapear o campo de relações entre população e meio ambiente e, consequentemente, colaborar para a estruturação de um desenvolvimento sustentável.

De forma fluida e estruturada, é dentro desta conceituação que Hogan vem explicitar a conexão de Sustentabilidade e DS com a demografia espacial. $\mathrm{O}$ autor sugere que para ser útil, o conceito de CS deve ser usado no âmbito multiescalar, com foco na escala de ecossistemas e que a sua utilização faça sentido do ponto de vista da organização social de um dado território. Ressaltando que não se trata de uma fórmula miraculosa, na qual será obtido um resultado fixo final, deve-se considerar o conceito de CS como uma matriz de possibilidades. O objetivo principal da CS sob este prisma da demografia espacial é tornar explícitas as relações entre as formas de organização social e os ecossistemas de um dado território, de um dado espaço geográfico.

Sendo assim, é importante destacar a conceituação de ecossistema aqui adotada, de acordo com Hogan (1993) e Berkes et. al (2002): sistema no qual vivem os seres humanos em interação com outros seres vivos e em contato com características físicas, químicas e biológicas do ambiente, em um contexto de unidade natural. Trata-se de uma interação mútua de processos ecológicos e sociais, onde a conjunção de eventos sociais e ambientais podem 
proporcionar a preservação e equilíbrio dos ecossistemas ou degradá-los.

Para a interação de processos ecológicos e sociais, Hogan (1993) chega a citar o termo socioecossistema, o qual considera uma expressão redundante, mas, é usada por ele em seu texto para chamar atenção para a componente social dos ecossistemas, sendo uma componente frequentemente reduzida ou ignorada por estudiosos ambientalistas. Neste trabalho, o termo socioecossistema será adotado, mesmo que o autor o tenha considerado uma tautologia.

\subsection{A análise multiescalar da demografia espacial pode viabilizar a práxis da Sustentabilidade e do Desenvolvimento Sustentável (DS)}

Com base em Hogan (1993), o debate sobre a CS de distintos ecossistemas dentro de um respectivo espaço geográfico pode contribuir para uma avaliação dos ganhos e perdas envolvidos em uma política de desenvolvimento. Isto porque a diversidade regional torna necessário um planejamento espacial específico. Assim, a unidade de análise a nível socioecossistêmico é indicada pelo autor como ideal para ser trabalhada dentro da Sustentabilidade e do DS, considerando, contudo, as suas respectivas relações e influências com o contexto regional e nacional.

No que se refere aos níveis escalares, entende-se que: escala local se refere a recortes espaciais em graus variáveis que permitam a possibilidade de uma vivência social e política intensa do espaço; escala mesolocal refere-se a níveis ecossistêmicos, geralmente colocados como referência de escala municipal; e escala regional caracteriza espaços de implementação de políticas públicas (IBGE,1999; SOUZA, 2013).

As escalas espaciais estão, portanto, vinculadas com o ambiente e sua respectiva CS e, como apontado por Hogan, uma análise multiescalar permite a compreensão adequada tanto da CS quanto da relação P-A em determinado espaço geográfico. No caso do Brasil, nota-se que muitos programas e políticas gover- 
namentais não realizaram este tipo de abordagem e resultaram no agravamento de quadros de vulnerabilidades social e ambiental. Tal insucesso se deve, principalmente, pela aplicação de programas e políticas que desconsideram o viés multiescalar dos territórios e que ignoram os aspectos de CS locais e regionais, considerando unidimensionalmente as necessidades e capacidades nacionais, executando-as em uma escala regional - e até mesmo, local.

Exemplos destas malogradas políticas e programas de desenvolvimento unidimensionais são encontradas no período da ditadura militar no Brasil (1964-1985), o qual foi marcado pela implementação dos principais programas de desenvolvimento econômico para o Cerrado, o POLOCENTRO e o PRODECER, (CARVALHO, 2019). Devido a sua completa negação das dinâmicas locais, regionais e, principalmente, dos aspectos físicos ambientais, os programas foram voltados para o desenvolvimento agrário, com de caráter predatório do meio ambiente, buscando intensificar a produção agrícola. Como consequência, nestas áreas do Cerrado onde os programas foram efetuados, a degradação ambiental se intensificou consideravelmente, além de instensificar as disparidades econômicas e sociais dessas regiões (CARVALHO, 2019).

É evidente, portanto, que ignorar ou negligenciar aspectos sociais, econômicos e ambientais em escalas locais e regionais é, para qualquer tentativa de desenvolvimento, sinônimo de injustiça social e degradação ambiental. Como apontado por Hogan (1993), a CS e, consequentemente, o DS de um determinado território deve ser analisado e aplicado em escalas menores, considerandose concomitantemente as suas relações a nível regional e nacional. Para exemplificar tal práxis multiescalar da Sustentabilidade e do DS que obtenha bons resultados e evite modelos programáticos heterônomos, Hogan (1993) ressalta como ponto de partida o nível dos socioecossistemas e, para isso, nos fornece um exemplo de unidade escalar adequada para estudos e planejamentos relacionados aos recursos hídricos, que seria a mesoescala das bacias hidrográficas. A unidade escalar da bacia hidrográfica é considerada uma escolha estratégica para a observação e a aná- 
lise das relações sociodemográficas-ambientais considerando-se a temática dos recursos hídricos.

Outra importante contribuição de Hogan no destaque unidade escalar e a sua respectiva relação com a Sustentabilidade é discutida no fenômeno da expansão urbana (urban sprawl). Em seu texto intitulado "Urban Sprawl: A Challenge for Sustainability" de 2008, o autor destaca que dependendo do enfoque dado a este fenômeno(local, regional, nacional ou internacional), pesquisadores e tomadores de decisão enfrentarão maior ou menor complexidade no que se refere a entender os desafios relacionados a ele - tais como poluição do ar, demanda por recursos hídricos, perda de espaços verdes, fragmentação espacial, e assim por diante. Hogan nos mostra ainda que, para enfrentar os desafios relacionados ao fenômeno da expansão urbana, e pensá-lo de forma sustentável, é necessário o uso de estudos espaciais combinados com pesquisas locais aprofundadas em cidades específicas para, enfim, propor soluções alinhadas com a Sustentabilidade e o DS.

Para Hogan, os principais desafios que devem ser solucionados caso o objetivo seja desenvolver cidades sustentáveis, estariam principalmente relacionados aos recursos naturais (por exemplo, o comprometimento da qualidade dos recursos hídricos), à relação entre a densidade populacional e a sua distribuição no espaço (por exemplo, a expansão de espaços urbanos não contíguos) e à mobilidade urbana relacionada ao aumento das distâncias em rotas diárias da população (por exemplo, a rota casatrabalho). Aqui temos, portanto, mais um exemplo de interação entre aspectos demográficos, espaciais e econômicos sociais: a distribuição da população em uma escala local e a relação que pode ser feita destes aspectos com a Sustentabilidade e DS.

Sendo assim, em termos práticos, é possível observar a associação entre demografia espacial, Sustentabilidade e DS em um número crescente de políticas e planejamentos urbanos, oriundas principalmente de iniciativas governamentais. A CS nesses projetos e planejamentos está vinculada de forma a auxiliar a estruturar a capacidade de desenvolvimento econômico e social, permitindo o uso dos recursos naturais e promover a preservação ambiental, 
bem como alcançar o nível de qualidade de vida almejada pela população (VARGAS, 2013; FERREIRA, 2019). No caso dos centros urbanos, por exemplo, são crescentes as formulações de políticas estratégicas baseadas em aspectos demográficos, econômicos e ambientais. Do Egito a Nova Iorque, temos exemplos de práticas de planejamento e desenvolvimento cidades verdes e ecológicas, que incluem a elaboração de uma estrutura urbana e planejamento de uso do solo e de seus sistemas de abastecimento de forma sustentáveis, considerando também a Sustentabilidade nos campos econômico e social (HOSAM et. al., 2016; CITY OF NEW YORK, 2007).

Teríamos, desta forma, um diálogo, trocas e transformações constantes entre espaço-geográfico e dinâmicas demográficas. A CS está diretamente vinculada com o espaço e o meio ambiente e, para proporcionar a otimização dessa capacidade, devemos levar em consideração os contextos multiescalares envolvidos (local, regional, nacional, internacional, ou, cidade, estado, país e globo). Em constante enriquecimento teórico e prático, o campo da Demografia Espacial se mostra capaz de abarcar características demográficas de uma população e, ao mesmo tempo considerar o seu contexto espacial para entender relações sociais, econômicas e políticas.

\section{Conclusão}

Os conceitos de Sustentabilidade e DS no decorrer das últimas décadas foram moldados para se adequar a uma ampla gama de orientações políticas e econômicas. Ao mesmo tempo, de forma lenta e gradativa, foi construído um núcleo de diretrizes ambientais, sociais, econômicas e políticas dentro destes conceitos, principalmente por órgãos internacionais. Atualmente, para se conclamar sustentável, existe a necessidade de se comprovar o cumprimento de algumas destas diretrizes, tais como preservação ambiental, desenvolvimento socioeconômico e políticas de equidade social. Entretanto, esses conceitos se apresentam ainda 
dinâmicos e em constante adequação, principalmente quando aplicados na dimensão espacial.

No presente trabalho discutimos a contribuição de Hogan para se pensar a relação entre Sustentabilidade, Desenvolvimento Sustentável e a Demografia Espacial. O autor contribuiu para as análises holísticas da relação População-Ambiente, principalmente por incorporar a dimensão espacial aos temas de Sustentabilidade, DS e os estudos demográficos. A demografia espacial contempla aspectos ambientais, sociais, econômicos e demográficos e, portanto, o espaço geográfico não estaria dissociado das dinâmicas populacionais. A relação entre ambiente, população e sociedade tem se mostrado importante para se pensar políticas públicas, planos governamentais e atividades econômicas que contemplem as diretrizes de Sustentabilidade e DS. As análises multiescalares, como a socioecossistêmica apresentada por Hogan, leva em consideração o contexto espacial em que esta escala mesolocal está inserida, exemplificando ainda que a capacidade de suporte de um determinado espaço geográfico não deve ser considerada apenas pelo viés ambiental, mas de acordo com o entendimento social que se tem deste espaço.

Sobre a formulação de uma agenda comum da Sustentabilidade e DS, como assim proposta por Hogan (1993), esta pode atualmente ser vista, por exemplo, no crescente uso das agendas internacionais de Sustentabilidade e DS - tais como as das Nações Unidas - em políticas públicas e planejamentos territoriais. Porém, essas agendas internacionais são tidas como referência e, a partir delas, políticas públicas, ações e planejamentos são propostos de forma que contemple as características geográficas, sociais, econômicas e políticas em questão.

A relação ambiente, população e sociedade apresenta desafios em diferentes escalas: local, regional, nacional e global. As agendas de Sustentabilidade e DS devem ser, portanto, ajustadas às realidades, necessidades e CS locais, para que, desta forma, políticas e iniciativas nestes temas sejam bem-sucedidas. Portanto, concluímos que não temos uma definição rígida de agendas nestes temas. 
Desta forma, os estudos e a iniciativa de Hogan de dar destaque e importância para a componente espacial nas investigações demográficas nos permitem e incentivam a dar sequência no seu legado, almejando soluções e propostas no campo de População-Ambiente, Sustentabilidade e DS que não sejam rígidas ou determinísticas. Observa-se que a contribuição e inovação da demografia espacial para estes temas reside na capacidade de conectar e promover uma análise multiescalar, e isto pode se tornar um possível facilitador para se pensar em diretrizes programáticas e iniciativas sustentáveis em diferentes realidades espaciais, demográficas, sociais e econômicas.

\section{Referências}

ORGANIZAÇÃODASNAÇÕESUNIDAS-ONU.The Sustainable Development Report 2021. Retrieved from United Nations General Assembly: https://sdgindex.org/reports/sustainable-developmentreport-2021/

BERKES, F.; COLDING, J.; FOLKE, C. Navigating SocialEcological Systems: Building Resilience for Complexity and Change. Cambridge University Press, Cambridge, 2002.

CARVALHO, J. P. S. Os Programas de Desenvolvimento Agrícola para incorporação do Cerrado no processo produtivo do Brasil. Revista de Economia da UEG - vol. 15, n. 1, jan/jun. 2019.

CITY OF NEW YORK. PlaNYC 2030 - A Greener, Greater New York, City of New York, United States, 2007.

DE CASTRO, M. C. Spatial Demography: An Opportunity to Improve Policy Making at Diverse Decision Levels. Popul Res Policy Rev 26, 477-509 (2007). https://doi.org/10.1007/s11113-0079041-x

FERREIRA, C. F. Modelagem da capacidade de suporte da área central de Sete Lagoas, MG [manuscrito] : subsídios para gestão urbana - 2019. 
GONÇALVES, V. J. Mudanças do governo no meio ambiente serão analisadas pelo novo Congresso. Senado Notícias, 01 de fev. 2019. Disponível em: https://www12.senado.leg.br/noticias/ materias/2019/01/31/mudancas-do-governo-no-meio-ambienteserao-analisadas-pelo-novo-congresso-1. Acesso em: 25 fev. 2021.

HOGAN, D.J.; OJIMA, R. Urban sprawl: a challenge for sustainability. In: MARTINE, G.; et al. (eds.) The New Global Frontier: urbanization, poverty and environment in the 21st century. London: Earthscan, 2008. p. 203-216.

HOGAN, D. J. Crescimento demográfico e meio ambiente. Revista Brasileira de Estudos de População, v. 8, n. 1/2, p. 61-71, 1991.

HOGAN, D. J. Crescimento populacional e desenvolvimento sustentável. Lua Nova, São Paulo, n. 31, p. 57-78, dez. 1993.

HOGAN, D. J.; D’ANTONA, Á. de O.; CARMO, R. L. do. Dinâmica Demográfica Recente da Amazônia. In: BATISTELLA, M.; MORAN, E.; ALVES, D. S. (Org.). Amazônia: natureza e sociedade em transformação. São Paulo: Edusp - Editora da Universidade de São Paulo, 2008, p. 71-116.

EL GHORAB, H. K.; SHALABY, H. A. Eco and Green cities as new approaches for planning and developing cities in Egypt. Alexandria Engineering Journal, v. 55, n. 1, p. 495-503, 2016.

HOVE, H. Critiquing Sustainable Development: A Meaningful Way of Mediating the Development Impasse? Undercurrent, v. 1, n. 1, p. 48-54, 2004.

INSTITUTO BRASILEIRO DE GEOGRAFIA E ESTATÍSTICA - IBGE. Noções básicas de cartografia I Departamento de Cartografia. Manuais técnicos em geociências. Rio de Janeiro: IBGE, n.8, 1999. 130 p.

INSTITUTO NACIONAL DE METROLOGIA, NORMALIZAÇÃO E QUALIDADE INDUSTRIAL e INSTITUTO BRASILEIRO DE DEFESA DO CONSUMIDOR - IDEC. Meio Ambiente e consumo. DF: INMETRO/IDEC, Coleção Educação para o consumo Sustentável, 2002. 
INTERNATIONAL GEOSPHERE-BIOSPHERE PROGRAMME - IGBP. A study of global change. In: International Council of Scientific Unions. Report n. 27. Stockolm, 54 p., 1993.

JOHNSTON, P.; et al. Reclaiming the definition of sustainability. Environ Sci Pollut Res Int. 2007 Jan; v. 14, n. 1, p. 60-66. doi:10.1065/ espr2007.01.375.

KLINK, C. A..; MACHADO, R. A conservação do cerrado. Megadiversidade, v 1, n. 1, p. 147-155, 2005.

MARANDOLA JR., E.; HOGAN, D. J. Em direção a uma demografia ambiental? Avaliação e tendências dos estudos de população e ambiente no Brasil, Revista Brasileira de Estudos de População, São Paulo, v. 24, n. 2, p. 191-223, jul./dez. 2007.

MARTINE, G. (Org.). População, meio ambiente e desenvolvimento: verdades e contradições. Campinas, SP: Editora da UNICAMP, 1993.

RATTNER, H. Sustentabilidade - uma visão humanista. Ambiente \& Sociedade. Campinas, n. 5, p. 233-240, dec. 1999.

SANTOS, M. A natureza do espaço. São Paulo: Hucitec, 1996. $392 \mathrm{p}$.

SARTORI, S.; LATRONICO, F.; CAMPOS, L. M. de S. Sustentabilidade e desenvolvimento sustentável: uma taxonomia no campo da literatura. Ambiente \& Sociedade, São Paulo, v. 17, n. 1, p. 01-22, mar. 2014.

SOUZA, M. L. de. Os Conceitos Fundamentais da Pesquisa SócioEspacial. Rio de Janeiro, Bertrand Brasil, 2013. 319p.

ORGANIZAÇÃO DAS NAÇÕES UNIDAS - ONU. Sustainable Development Goals: 17 Goals to transform our world. [Online]. New York, 2015. Disponível em: https://www.un.org/ sustainabledevelopment/sustainable-development-goals/. Acesso em: 30 março 2021. 
As conexões entre Sustentabilidade e Desenvolvimento...

VARGAS, G. P. de. Capacidade de suporte para adensamento urbano [manuscrito]: proposta de análise multicritério para avaliação / Guilherme Pereira de Vargas. - 2013

VOSS, P. Demography as a spatial social science. Population Research and Policy Review, v. 26, p. 457-476, set. 2007. 